\title{
The Task-Evoked Pupil Response in Divided Auditory Attention Tasks
}

DOI: $10.3766 /$ jaaa. 17060

\author{
Jennifer Baldock* \\ Sarosh Kapadia* \\ Willem van Steenbrugge*
}

\begin{abstract}
Background: Successful processing of complex auditory information relies on the interplay between low-level sensory processing and higher-level cognitive processing. However, the extent to which specific auditory processing tasks rely on cognitive processing as opposed to lower-level sensory processing is unclear. The task-evoked pupil response (TEPR) can quantify the cognitive load that complex listening tasks elicit. Previous research by Koelewijn et al (2014) indicated that dividing attention across two sentences presented dichotically resulted in larger pupil dilation (indicative of greater cognitive load) compared with selectively attending to one. However, it was unclear whether the larger pupil dilation measured during the divided attention task were the result of dividing attention or were due to the increased memory demand inherent to that task.
\end{abstract}

Purpose: The first aim of the current study was to address the above issue of memory demand by comparing pupil dilation between divided and selective auditory attention tasks, while keeping memory and response load constant. The second aim was to further clarify the influence of memory demands on TEPRs in these auditory tasks by comparing the pupil dilation recorded to measures of participants' digit memory capacity.

Research Design: A repeated measures design was used. Each participant undertook two selective and three divided auditory attention tasks, generated by varying the specific instructions before each condition of the dichotic digits test (DDT). In addition, participants completed forward and reverse digit span (DS) tasks.

Study Sample: Thirty-one otologically healthy adults (aged 18-40 years) participated in this study.

Data Collection and Analysis: A repeated measures analysis of variance was used to compare mean and peak pupil dilation between the selective and divided attention tasks. Spearman correlation analyses were used to examine potential relationships between DS scores and mean and peak pupil dilation elicited by the DDT conditions.

Results: Participants demonstrated larger mean and peak pupil dilation (indicative of greater cognitive load) when they were required to divide their attention across both ears than when they were required to selectively attend to input in one ear. DS scores were not significantly correlated with mean or peak pupil dilation measures.

Conclusions: Auditory divided attention tasks involve significantly greater cognitive load than auditory selective attention tasks, even when memory demands are equal. In addition, mean and peak pupil dilation generated during the DDT are not significantly associated with digit memory capacity. The findings indicate that poor performance on tasks involving divided attention may be due to a cognitive deficit as opposed to an auditory processing deficit. Clinicians should consider this when using divided attention tasks in auditory processing assessments.

Key Words: cognitive load, dichotic listening, divided attention, memory demands, selective attention, task-evoked pupil response

*Flinders University, Adelaide, South Australia, Australia

Corresponding author: Jennifer Baldock, Adelaide, South Australia 5001, Australia; Email: bald0106@uni.flinders.edu.au

Presented in part at AudiologyNOW!, Phoenix, AZ, April 2016, and Audiology Australia National Conference, Melbourne, Australia, May 2016. 


\begin{abstract}
Abbreviations: ANOVA = analysis of variance; $A P D=$ auditory processing disorder; DDT = dichotic digits test; DDTALL = dichotic digits test "All" condition; DDTFP = dichotic digits test "first pair" condition; DDTLE = dichotic digits test "left ear" condition; DDTLP = dichotic digits test "last pair" condition; DDTRE = dichotic digits test "right ear" condition; DS = digit span; LC = locus coeruleus; TEPR = task-evoked pupil response
\end{abstract}

\section{INTRODUCTION}

$\mathrm{L}$ istening in ideal conditions is a relatively effortless task. However, listening may become more effortful in conditions where the quality of the signal is degraded, for example, in the presence of background noise. During such complex listening conditions, individuals must distinguish between and select the relevant auditory signals, while simultaneously filtering out irrelevant signals, the so-called "cocktail party effect" (Cherry, 1953). In addition, individuals are often required to divide their attention between two or more signals of interest. Listening in such conditions can increase the cognitive load that is required to understand and generate appropriate responses to speech (Koelewijn et al, 2014) and can result in listening-related fatigue (McGarrigle et al, 2014). Cognitive load has been defined as "any factor placing unusually high demands on central attentional and mnemonic capacities" (Mattys et al, 2012). Attention, working memory (and short-term memory), and inhibitory control are commonly referred to as executive functions. These functions operate together to maintain and process relevant information related to the task at hand and to inhibit irrelevant information with reference to a predetermined goal (Diamond, 2013). Thus, when there is more than one auditory signal present, more cognitive load will be imposed on the individual because attention and memory functions will have more auditory information to process.

Before cognitive processing, auditory information must travel up the auditory nerve through multiple neural networks to the auditory cortex. Auditory processing refers to the neurobiological activity in the central auditory nervous system that underlies perceptual processing of auditory information (ASHA, 2005). During the early stages of auditory processing, acoustic stimuli are divided into streams based on sensory and physical components (Bregman, 1994). The early stages of processing aid later cognitive processing.

Poor auditory processing can result in academic and communication difficulties but can also have adverse consequences for psychosocial wellbeing (ASHA, 2005). When an individual experiences difficulty with one or more auditory processing abilities, despite having a normal audiometric test result, they may be diagnosed with an auditory processing disorder (APD). ASHA (2005) defined APD as being the result of low-level, neural processing deficits in the auditory system and asserted that APD is not the result of higher-order, cognitive deficits. However, as argued by Moore (2015), one of the fun- damental questions concerning APD is whether listening difficulties are the result of sensory, cognitive, or both sensory and cognitive impairments. An objective measure of cognitive load may shed light on the degree to which cognitive factors can influence performance during auditory processing tests.

Pupil dilation, as measured by the task-evoked pupil response (TEPR), has been used as an objective measure of cognitive load in a variety of applications and can provide information about cognitive load that is not reflected in performance (Beatty and Lucero-Wagoner, 2000). Evidence suggests a strong link between taskevoked pupillary changes and activation of the locus coeruleus (LC). The sympathetic activity that innervates the dilator muscles in the iris receives excitatory projections from the LC via norepinephrine projection (Einhäuser, 2017). Norepinephrine is a neurotransmitter associated with emotions, arousal, and executive function (Aston-Jones and Cohen, 2005; Benarroch, 2009; Gilzenrat et al, 2010; McGarrigle et al, 2014; Sirois and Brisson, 2014). The LC is also involved with inhibiting parasympathetic constriction at the EdingerWestphal nucleus. Thus, the LC contributes to the mediation of the cognitive pupil dilation, directly via the sympathetic pathway and indirectly via inhibition of the parasympathetic pathway (Einhäuser, 2017). However, despite such convincing evidence for the relationship between LC activation and cognitive pupil dilation, direct physiological evidence is lacking. Thus, the mechanisms underlying this relationship are not clearly understood.

Hess and Polt (1964) were among the first to use pupillometry as a measure of cognitive load. They demonstrated that more difficult mental arithmetic problems (e.g., $16 \times 23$ ) produced greater pupil dilation than more simple arithmetic problems (e.g., $7 \times 8$ ) and noted that dilation occurred after a stimulus was presented (and during processing) and constriction occurred only after responses had been given. Kahneman and Beatty (1966) demonstrated that TEPRs are sensitive to short-term auditory memory tasks (strings of 3-7 digits presented for immediate recall). Furthermore, they observed that the pupil dilation increased as a function of digit string length (i.e., the seven digit string produced the largest pupillary dilation).

Subsequently, a number of researchers have used TEPRs to study cognitive load in various listening conditions (Kramer et al, 1997; 2013; Zekveld et al, 2010; 2011; Kuchinsky et al, 2013; Winn et al, 2015). Kramer et al (1997) examined pupil dilation during a speech 
reception in noise task while manipulating the signal to noise ratio. They found that pupil dilation was smaller in more favorable signal to noise ratio conditions and larger for less favorable conditions. Thus, TEPRs are also sensitive to variations in listening conditions. More recently, Kramer et al (2013) examined TEPRs in response to a variety of auditory tasks including the following: passive listening, auditory detection of meaningless target noise bursts in noise, and identification of meaningful words in noise. They found that the mean pupil dilation was significantly larger for the condition in which participants had to identify meaningful words than for passive listening and for the detection of noise bursts in noise. They observed no significant differences in mean pupil dilation between the passive listening condition and the detection of noise bursts in noise condition. Therefore, they concluded that language tasks might involve a higher degree of cognitive effort to complete than nonlanguage tasks. Thus, when investigating auditory processing abilities, it may be beneficial to use tasks with minimal linguistic load, as they require less cognitive effort and may be more sensitive to lower-level auditory processes.

Dichotic listening tasks represent an important class of auditory processing assessments. These tasks involve the simultaneous presentation of a different auditory stimulus to each ear (Broadbent, 1971). Many types of dichotic listening tasks have been used in APD test batteries, including the dichotic digits test (DDT), staggered spondaic word test, competing words test, and competing sentences test. They are used to test either binaural integration, that is, the ability to correctly attend to both competing signals in the two ears (divided attention) or binaural separation, that is, the ability to attend to an auditory signal in one ear while ignoring a different signal in the other ear (selective attention) (Schow et al, 2000; ASHA, 2005).

Koelewijn et al (2014) investigated the effects of divided attention on the pupil response during a dichotic sentence task. Participants were required to attend to and repeat back either one or both of two dichotically presented sentences. They found that dividing attention across two sentences presented dichotically (compared with selectively attending to one) resulted in significantly greater pupil dilation. Therefore, Koelewijn et al (2014) concluded that divided attention during dichotic tasks resulted in more cognitive load than selective attention. This conclusion is of importance, given the widespread use of dichotic testing in auditory processing assessments. It suggests that any apparent deficit in dividing attention, across both ears in particular, may reflect a deficit in high-level cognitive factors, rather than in low-level auditory processing factors.

Although Koelewijn et al (2014) concluded that the TEPR was specifically sensitive to divided attention, the nature of the tasks used (focus on and repeat two sentences versus one sentence) resulted in some uncertainty regarding this conclusion. The divided attention condition contained double the amount of information to be processed, stored, and recalled than the selective attention condition, leading to an increased demand on memory. As previously stated, Kahneman and Beatty (1966) showed that TEPRs are sensitive to variations in memory load. Therefore, it was unclear whether the larger pupil dilation observed in the divided attention condition was purely the result of dividing attention between two sentences or whether it was the result of the increased memory demands in this condition. Koelewijn et al (2014) mentioned this issue but asserted that the pupil dilation recorded during divided attention was still considerably larger than pupil dilation recorded in their previous research, when sentences were presented with different masker types (Koelewijn et al, 2012). However, this explanation does not directly address whether the increased memory demands in the divided attention condition in the study by Koelewijn et al (2014) was partly or wholly responsible for the larger pupil dilation in this condition than in the selective attention condition. This is an important point that warrants further investigation to enhance our understanding of the cognitive load that particular auditory processing tasks may elicit.

The current research was concerned with how the TEPRs in dichotic tasks are affected by divided versus selective attention when memory demands are held constant. The standard DDT (Musiek, 1983) involves the simultaneous presentation of two different digits to each ear. It was chosen for this study because it could be readily applied in a manner that equalized the amount of material for recall between divided attention and selective attention conditions. In addition, it is also expected to be less subjected to the effects of extraneous language-related factors than other word- or sentencebased dichotic tasks. To date, there have not been any reports of the cognitive load associated with the DDT using pupillometry. However, Parkinson (1974) investigated cognitive factors associated with performance on a dichotic digits task and found that digit span (DS) performance accounted for $79.4 \%$ of the variance in dichotic digits performance when participants were required to recall eight dichotic digits. This strong association between Parkinson's dichotic digits task performance and DS performance may suggest that the short-term memory demands inherent to the standard DDT may significantly contribute to the overall cognitive load measured in the test. In addition, Kahneman and Beatty (1966) documented that the TEPR is sensitive to memory demands, observing greater pupil dilation as DS length was increased. Thus, it is possible that the TEPRs elicited in the DDT might also be sensitive to an individual's DS because of the memory demands inherent in the DDT. 
The primary aim of the current study was to investigate whether the TEPR is specifically sensitive to divided auditory attention or whether varying memory demands may have contributed to the conclusion in the previous literature (Koelewijn et al, 2014). This was pursued by measuring TEPRs during divided and selective auditory attention tasks, with constant memory and response load. The secondary aim was to examine whether TEPRs obtained during the DDT are related to participants' digit memory capacities. This could improve the understanding of the cognitive processes evoked during DDT performance.

\section{METHODS}

\section{Participants}

Participants were 31 otologically normal adults aged 19-39 years $(M=26$, standard deviation $=4.72)$ with no reported listening, speech, language, attention, or learning difficulties. An estimation of sample size/ power analysis (confidence 0.95 , medium effect size) was conducted for the repeated measures analysis of variance (ANOVA) using the G Power (3.0) package (Faul et al, 2007) showing a sample size of 31 had a 95\% chance of correctly rejecting the null hypothesis. Participants were recruited via flyers and personal contacts. All participants had pure tone hearing thresholds $\leq 20 \mathrm{~dB}$ HL at all octave frequencies between 250 and $8000 \mathrm{~Hz}$ in each ear and normal (Jerger Type A) tympanograms. This study was approved by the Southern Adelaide Clinical Human Research Ethics Committee.

\section{Procedure}

All testing took place in a sound-treated booth. Audiometry and auditory testing were conducted using the MedRx system and ER-3A insert earphones. The SensoMotoric Instruments Eye Tracking Glasses 2.0 (Germany) and SensoMotoric Instruments iView software was used to record TEPRs at a sample rate of $60 \mathrm{~Hz}$. An A4-sized checkerboard was used to perform a three-point software calibration of the eye tracking glasses for each participant. This was placed approximately at eye level, a distance of $\sim 1.5 \mathrm{~m}$. The calibration checkerboard was replaced by a white sheet with single black dot in the center $(24 \mathrm{~mm}$ diameter), and participants were instructed to look at the dot during testing to minimize the risk of variations in pupil diameter due to shifts in focus. The ambient lighting level in the room was kept constant at 55 lux for all recordings.

The DDT (Musiek, 1983) involved the simultaneous presentation of two digits (1-10, excluding 7), to each ear (total of four digits). Each participant undertook three divided and two selective auditory attention tasks generated by varying the instructions of the DDT as given below:
- Divide attention between left and right ear input and repeat back all four numbers (DDTALL).

- Divide attention between left and right ear input and repeat back the first pair of digits only (DDTFP).

- Divide attention between left and right inputs and repeat back the last pair of digits only (DDTLP).

- Selectively attend to and repeat back the left ear input only (DDTLE).

- Selectively attend to and repeat back the right ear input only (DDTRE).

Each condition used identical stimuli and only the task instruction varied between conditions. The design of the four additional conditions ensured that there was an equal amount of materials for recall between the divided attention conditions (first and last pair) and the selective attention conditions (left and right ear), that is, two digits to be encoded and recalled from memory. There were ten trials for each condition, and participants received a score of 1 for every correct response (total score out of 10). Preliminary data demonstrated that ten trials of the DDT conditions were sufficient to elicit robust TEPRs.

The Audacity audio editor software was used to set the length of each DDT trial to 10 sec by increasing the length of the silent intervals between trials as needed. The original content of the DDT recording was otherwise unchanged. DDT stimuli were presented at $50 \mathrm{~dB}$ HL via foam tip insert earphones.

Digit memory capacity was assessed using the forward and reverse DS tests from the Test of Auditory Processing Skills-3 (Martin and Brownell, 2008). The sequence length was increased by one digit after two correct responses on trials of the same length, as per the test manual. Performance was scored according to the Test of Auditory Processing Skills-3 protocol. DS stimuli were presented at a comfortable listening level.

Test order and condition order were counterbalanced using a Latin square design to avoid any order effects.

\section{Processing of Pupil Data}

Pupil data were initially processed using the DADiSP signal analysis software. Blink artefacts were identified by first removing all values within a trace where pupil diameter was reported as $0 \mathrm{~mm}$ and then removing all values that were less than three standard deviations below the mean of the resulting pupil trace. Segments of the traces that were identified as blinks were replaced by linear interpolation from four points to the left of the blink to eight points to the right of the blink. Furthermore, all traces were examined visually within DADiSP (Data Analysis and Display), and the blink threshold was adjusted downward if necessary to prevent clipping of traces. Any traces with $>15 \%$ blink values (which amounted to $7 \%$ of all the traces in the study) were 
excluded from further analyses. The remaining traces for a given condition and participant were averaged and smoothed using a five-point moving average filter. Before peak identification and calculation of dilation measures, these traces were further smoothed using a 20-point moving average filter. Pupil data were analyzed for the left eye only.

Pupil diameter at the 2-sec point, that is, at the onset of the stimulus, was taken as the baseline for the dilation measures. For each averaged and smoothed trace, peak dilation was defined as the largest diameter value (relative to the baseline) within the interval from 2 to 8 sec. The mean pupil dilation was defined as the mean dilation (relative to the baseline) within the interval from 2 to 8 sec. Thus, all elements of the standard DDT are included in the dilation measurement epoch, including unprompted verbal recall of digits.

\section{Data Analyses}

All statistical analyses were completed with SPSS version 21 software (IBM Corp., Armonk, NY). Repeated measures ANOVA were used to determine if selective and divided attention conditions significantly affected peak and mean pupil dilation. Multiple comparisons with a Bonferroni-adjusted alpha value were used to identify significant differences in mean and peak pupil dilation between DDT conditions where the ANOVA indicated significant effects.

Kolmogorov-Smirnov tests indicated that the raw data for DS forward and reverse were not normally distributed. Spearman correlations were computed to examine the relationship between DS (forward and reverse) raw scores and mean and peak pupil dilation in all conditions of the DDT.

\section{RESULTS}

A s expected for this sample, the DDT conditions elicited a strong ceiling effect in performance. Across all participants and all DDT conditions, only $1.3 \%$ of data indicated a performance below 8/10.

Figure 1 shows pupil responses averaged over all participants for each DDT condition (DDTALL, DDTLE, DDTRE, DDTFP and DDTLP). All conditions elicited marked changes to pupil diameter during the 10 -sec trial period, in response to the stimuli.

Figure 2 shows the average peak dilation across the five DDT conditions. A repeated measures ANOVA was conducted to compare peak dilation for the five DDT conditions (DDTLE, DDTRE, DDTFP, DDTLP and DDTALL,). Mauchly's test of sphericity indicated that the sphericity assumption had not been violated $\left[\chi^{2}(9)=13.52, p=0.141\right]$. Thus, sphericity-assumed values were used. There was a significant effect for DDT condition, $F_{(4,120)}=14.78$, $p<0.001, \eta_{\mathrm{p}}{ }^{2}=0.33$, showing that DDT condition had a substantial effect on peak pupil dilation.

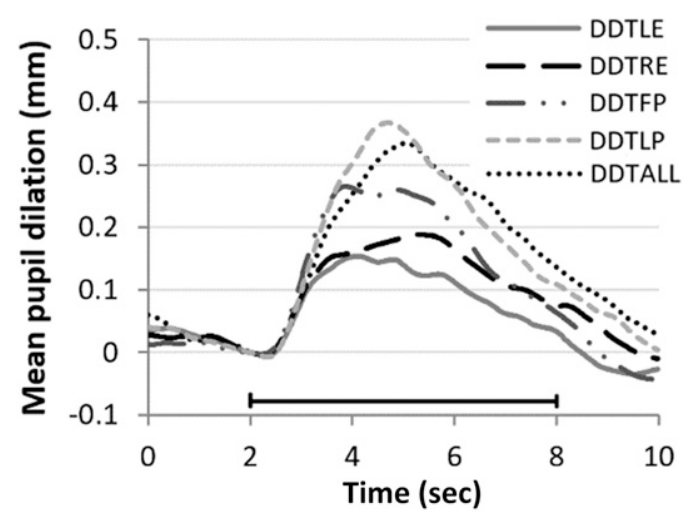

Figure 1. Pupil responses for the five DDT conditions averaged over participants. The onset of stimulus was at 2 sec. Divided attention conditions (DDTFP, DDTLP, and DDTALL) produced greater dilation than selective attention conditions (DDTLE and DDTRE). The horizontal line (from 2 to $8 \mathrm{sec}$ ) indicates the time window for calculating peak and mean pupil dilation (presented in Figures 2 and 3). Mean standard error values across all participants were as follows: DDTALL: 0.019, DDTLE: 0.019, DDTRE: 0.017, DDTFP: 0.019, and DDTLP: 0.021 .

Multiple comparisons revealed that peak pupil dilation in DDTFP, DDTLP, and DDTALL was significantly larger than peak pupil dilation in DDTLE and DDTRE. In addition, peak pupil dilation in DDTLE and DDTRE did not significantly differ from each other. DDTFP, DDTLP, and DDTALL did not significantly differ from each other (Table 1).

Figure 3 shows the average mean dilation for the five DDT conditions. A repeated measures ANOVA was conducted to compare mean dilation for all five conditions of the DDT (DDTLE, DDTRE, DDTFP, DDTLP, and DDTALL). Mauchly's test of sphericity indicated that the sphericity assumption had not been violated $\left[\chi^{2}(9)=14.87, p=0.141\right]$. Thus, sphericity-assumed values were used. There was a significant effect for DDT condition, $F_{(4,120)}=9.83, p<0.001, \eta_{\mathrm{p}}{ }^{2}=0.28$, showing that DDT condition had a large effect on mean pupil dilation.

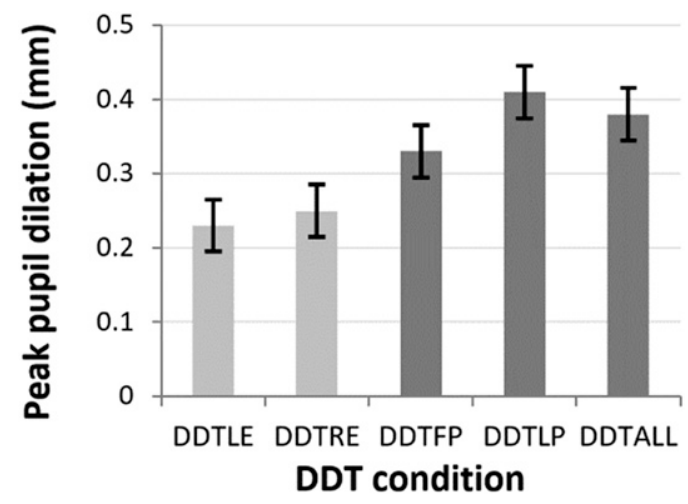

Figure 2. Mean values (standard error bars) across all participants for peak pupil dilation in the five DDT conditions. Peak pupil dilation was larger for divided attention conditions (DDTFP, DDTLP, and DDTALL) than it was for selective attention conditions (DDTLE and DDTRE). 
Table 1. Differences in Peak Pupil Dilation, Statistically Significant Differences in Bold (Column Heading-Row Heading: DDT Condition) ( $p$-Values Given in Parentheses as Defined by the Bonferroni Multiple Comparison Test)

\begin{tabular}{|c|c|c|c|c|c|}
\hline & DDTLE & DDTRE & DDTFP & DDTLP & DDTALL \\
\hline$\overline{\text { DDTLE }}$ & & $0.023(1.00)$ & $0.098(0.008)$ & $0.181(<0.001)$ & $0.149(<0.001)$ \\
\hline DDTRE & $-0.023(1.00)$ & & $0.076(0.033)$ & $0.158(<0.001)$ & $0.126(0.018)$ \\
\hline DDTFP & $-0.098(0.008)$ & $-0.076(0.033)$ & & $0.083(0.062)$ & $0.051(1.00)$ \\
\hline DDTLP & $-0.181(<0.001)$ & $-0.158(<0.001)$ & $-0.083(0.062)$ & & $-0.032(1.00)$ \\
\hline DDTALL & $-0.149(<0.001)$ & $-0.126(0.018)$ & $-0.051(1.00)$ & $0.032(1.00)$ & \\
\hline
\end{tabular}

Multiple pairwise comparisons revealed that mean pupil dilation in DDTFP, DDTLP, and DDTALL was significantly larger than in DDTLE. In addition, mean pupil dilation in DDTLP and DDTALL was significantly larger than in DDTRE. DDTLE and DDTRE did not significantly differ from each other. DDTFP, DDTLP, and DDTALL did not significantly differ from each other (Table 2).

Participants' performance on both the forward DS (range $=14-32$, median $=24$, interquartile range $=$ 4 ) and the reverse DS (range $=9-30$, median $=15$, interquartile range $=8$ ) tests were within the normal range for the sample (Martin and Brownell, 2008).

Tables 3 and 4 show Spearman rho correlation coefficients for DS forward and DS reverse scores compared with peak pupil dilation and mean pupil dilation (respectively) during the five DDT conditions (DDTLE, DDTRE, DDTFP, DDTLP, and DDTALL). Scores on the DS forward and reverse subtests were not significantly correlated with mean or peak dilation values in any of the DDT conditions.

\section{DISCUSSION}

$\mathrm{T}$ he primary aim of the present study was to compare cognitive load (as measured using TEPRs) between selective and divided auditory attention tasks when memory demands were held constant. Selective

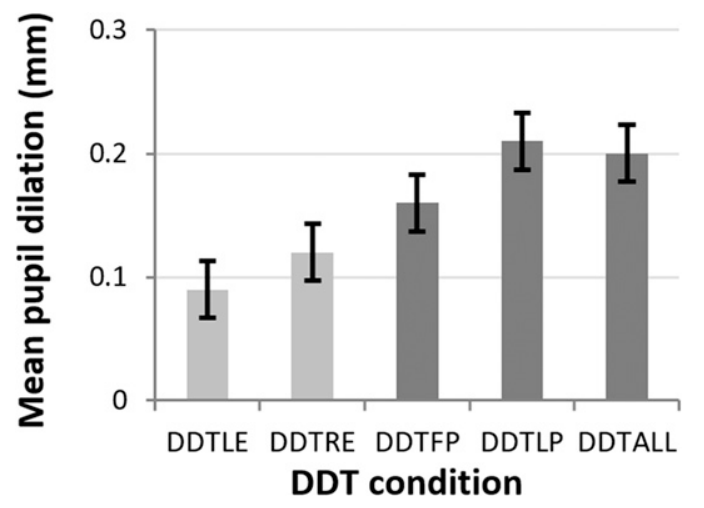

Figure 3. Mean values (standard error bars) across all participants for mean pupil dilation in the five DDT conditions. Mean pupil dilation was larger for divided attention conditions (DDTFP, DDTLP, and DDTALL) than it was for selective attention conditions (DDTLE and DDTRE). and divided auditory attention tasks were based on the DDT, as it could be readily applied in a manner that equalized memory demands, that is, by having an equal number of stimuli between conditions. Furthermore, the DDT is routinely used as a diagnostic tool in APD test batteries and is expected to be less subjected to the effects of linguistic knowledge and processes than other word- or sentence-based dichotic tasks. It was reasoned that the primary processes involved in undertaking these tasks would be selective or divided auditory attention and digit memory storage and recall.

As expected in adults with no reported listening or cognitive difficulties, participants responded correctly to most stimuli. This also indicated that participants were appropriately engaged in the conditions even though there were no significant differences in performance between the five DDT conditions.

Each DDT condition elicited marked changes in pupil diameter. In contrast to the DDT performance results, peak pupil dilation was significantly larger in DDTFP and DDTLP than peak pupil dilation in DDTLE and DDTRE. This showed that more cognitive load was associated with tasks that required divided attention (DDTFP and DDTLP) than tasks that required selective attention (DDTLE and DDTRE) with the same number of stimuli for recall.

A similar pattern of results was found with respect to mean pupil dilation. Mean pupil dilation was significantly larger in DDTFP and DDTLP than in DDTLE. Mean pupil dilation in DDTLP was significantly larger than mean pupil dilation in DDTRE. Thus, once again, more cognitive effort was necessary to perform tasks that required divided attention (DDTFP and DDTLP) than to perform tasks that required selective attention (DDTLE and DDTRE), with the exception of the pairwise comparison between DDTFP and DDTRE. The slight difference in the findings between peak and mean pupil dilation may reflect the possibility that peak dilation is a more sensitive measure of cognitive load, as dilation is not averaged over an extended period. This is congruent with previous results obtained by Zekveld et al (2010) who also observed greater differences in pupil dilation between background noise levels when peak dilation measures were used than when mean dilation measures were used. Conversely, peak measures seem to be more subjected to noise or random variations in dilation than mean measures are (Beatty and Lucero-Wagoner, 2000). 
Table 2. Differences in Mean Pupil Dilation, Statistically Significant Differences in Bold (Column Heading-Row Heading: DDT Condition) ( $p$-Values Given in Parentheses as Defined by the Bonferroni Multiple Comparison Test)

\begin{tabular}{|c|c|c|c|c|c|}
\hline & DDTLE & DDTRE & DDTFP & DDTLP & DDTALL \\
\hline DDTLE & & $0.032(1.00)$ & $0.071(0.007)$ & $0.115(<0.001)$ & $0.112(<0.001)$ \\
\hline DDTRE & $-0.032(1.00)$ & & $0.040(0.191)$ & $0.083(<0.001)$ & $0.081(0.031)$ \\
\hline DDTFP & $-0.071(0.007)$ & $-0.040(0.191)$ & & $0.044(0.322)$ & $0.041(0.665)$ \\
\hline DDTLP & $-0.115(<0.001)$ & $-0.083(<0.001)$ & $-0.044(0.332)$ & & $-0.003(1.00)$ \\
\hline DDTALL & $-0.112(<0.001)$ & $-0.081(0.031)$ & $-0.041(0.665)$ & $0.003(1.00)$ & \\
\hline
\end{tabular}

The significant difference between mean and peak pupil dilation for divided and selective attention tasks showed that the different conditions resulted in different amounts of cognitive load, even though the performance between conditions was not significantly different.

The present findings support those of Koelewijn et al (2014) who also concluded that TEPRs are specifically sensitive to divided auditory attention during speech processing. This was true for the current study, even when memory demands were held constant between the divided and selective attention tasks. In addition, the current findings suggest that TEPRs are also sensitive to divided auditory attention tasks with minimal linguistic load.

These findings indicate that divided auditory attention (binaural integration) tasks involve significant cognitive load for normal-hearing adults and suggest that a poor result on an auditory processing test involving divided attention may, in some instances, reflect higherorder cognitive deficits, rather than low-level sensory deficits. Individuals may perform poorly on divided attention tasks (and more poorly than on selective attention tasks) primarily because of a cognitive issue, not because of a deficit related to central auditory processing. However, this suggestion is somewhat speculative because it is yet to be confirmed whether similar results are obtained in individuals with listening difficulties.

The secondary aim of the current study was to investigate the relationship between DS and TEPRs elicited during DDT performance. Parkinson (1974) found that digit memory accounted for $79.4 \%$ of the variance in the performance on a DDT. Thus, it was expected in the present study that across participants, DS performance would be related to the TEPRs elicited by the DDT. However, participants' DS forward and reverse scores did not significantly correlate with mean or peak pupil dilation in any of the DDT conditions, indicating that cognitive load while performing the DDT tasks was not associated with digit memory capacity. This could be because of the relatively low number of digits to be recalled in our DDT tasks (two digits or, at most, four digits). The dichotic digits task used by Parkinson (1974) used four dichotic digits pairs (eight digits to be recalled), which would have placed a larger demand on memory. The Spearman correlations in the present study could support this conclusion, as the mean and peak pupil dilation recorded during the DDTALL condition (which involved the greatest number of digits) showed the largest association with forward and reverse DS scores (albeit, not significantly). Furthermore, Parkinson instructed their participants to attend to either the left or the right ear and ignore the other, but in the recall phase, participants were instructed to repeat back everything they heard (including the unattended stimuli, if possible). Thus, there were methodological differences between the present study and that of Parkinson (1974).

The current findings indicate that the cognitive load associated with performing the DDT is not related to individual digit memory capacity for young adults with no cognitive or listening difficulties. Digit memory capacity may be associated with the cognitive load experienced when undertaking the DDT in other populations, such as children or individuals with listening difficulties or APD. For example, using a sample of children, Tomlin et al (2015) found that auditory working memory (as measured by DS) was correlated with DDT performance. As the DDT is commonly used as part of a test battery to diagnose APD in children, it may also be beneficial to examine the relationship between the cognitive load

Table 3. Correlation Coefficients for Spearman Rho ( () ), DS Score vs. Peak Pupil Dilation

\begin{tabular}{|c|c|c|c|c|c|}
\hline & DDTLE & DDTRE & DDTFP & DDTLP & DDTALL \\
\hline \multicolumn{6}{|l|}{ DS reverse } \\
\hline$\rho$ & 0.21 & 0.01 & -0.10 & 0.17 & 0.34 \\
\hline$p$ value & $(0.250)$ & $(0.962)$ & $(0.579)$ & $(0.360)$ & $(0.062)$ \\
\hline $\mathrm{Cl}$ & $0.16-0.53$ & $0.35-0.36$ & -0.44 to 0.26 & -0.2 to 0.49 & -0.02 to 0.62 \\
\hline \multicolumn{6}{|l|}{ DS forward } \\
\hline$\rho$ & 0.07 & 0.06 & 0.00 & 0.09 & 0.17 \\
\hline$p$ value & $(0.710)$ & $(0.728)$ & $(0.990)$ & $(0.640)$ & $(0.347)$ \\
\hline $95 \% \mathrm{Cl}$ & -0.29 to 0.41 & -0.3 to 0.41 & -0.35 to 0.35 & -0.27 to 0.43 & -0.2 to 0.49 \\
\hline
\end{tabular}

$\overline{\mathrm{Cl}}=$ confidence interval. 
TEPR in Divided Auditory Attention Tasks/Baldock et al

Table 4. Correlation Coefficients for Spearman Rho ( $\rho$ ), DS Score vs. Mean Pupil Dilation

\begin{tabular}{|c|c|c|c|c|c|}
\hline & DDTLE & DDTRE & DDTFP & DDTLP & DDTALL \\
\hline \multicolumn{6}{|l|}{ DS reverse } \\
\hline$\rho$ & 0.28 & 0.14 & -0.09 & 0.04 & 0.31 \\
\hline$p$ value & $(0.133)$ & $(0.440)$ & (0.616) & $(0.814)$ & $(0.088)$ \\
\hline $95 \% \mathrm{Cl}$ & -0.08 to 0.58 & -0.23 to 0.47 & -0.43 to 0.27 & -0.32 to 0.39 & -0.05 to 0.6 \\
\hline \multicolumn{6}{|l|}{ DS forward } \\
\hline$\rho$ & 0.10 & 0.12 & 0.00 & 0.10 & 0.16 \\
\hline$p$ value & $(0.575)$ & $(0.531)$ & (0.998) & (0.958) & (0.397) \\
\hline $95 \% \mathrm{Cl}$ & -0.26 to 0.44 & -0.24 to 0.45 & -0.35 to 0.35 & -0.26 to 0.44 & -0.21 to 0.49 \\
\hline
\end{tabular}

associated with performing the DDT and individual digit memory capacity in a sample of children and/or individuals with APD.

Interestingly, the current results indicated that mean and peak pupil dilation in the DDTALL condition did not significantly differ from the mean and peak pupil dilation in the DDTFP and DDTLP conditions. This may be surprising because the DDTALL condition required divided attention and recall of all four digits (as opposed to two in DDTFP and DDTLP). This result may provide further evidence that the TEPR (at least for the standard DDT and for young adults with no cognitive or listening difficulties) is specifically sensitive to divided attention, as the additional memory demands in DDTALL did not have a significant impact on the results. However, this result may also be due to the additional factors that were inherent in the DDTFP and DDTLP and not DDTALL. For example, in these conditions, participants had to focus attention on the first or the last pair of digits, respectively. In DDTALL, participants had to simply repeat back every digit they heard. Thus, task demands may have differed between the conditions.

In addition, mean and peak pupil dilation measured in DDTLE and DDTRE did not significantly differ from each other. This indicated that there was no difference in cognitive load when attending to the right versus the left ear. Commonly, dichotic listening tasks reveal a performance advantage for linguistic stimuli presented to the right ear (Kimura, 1961; Bryden, 1988). However, mean and peak pupil dilation did not indicate a cognitive advantage for one ear over the other in the DDT used in the current study. This could be because the tasks were not difficult enough, for this participant sample, to show an associated cognitive advantage for DDTRE. However, in a preliminary report, Kan and Winn (2016) found evidence to suggest that attending to the right ear required less effort as indicated by the TEPR, even when performance scores were similar. It may be beneficial to measure TEPRs during a different dichotic task, or in a different sample of participants, where a performance advantage for the right ear is demonstrated to further investigate the relationship between ear advantage and cognitive load.
Several researchers have suggested that task-relevant, overt motor responses (e.g., verbal responses or clicking a response button) can augment TEPRs (Bradshaw, 1968; Simpson, 1969; Simpson and Hale, 1969). Kahneman et al (1968) investigated this effect and concluded that verbal response requirements increase the cognitive load associated with tasks but that this increase is validly reflected in the measure of the load. The standard administration of the DDT requires participants to provide verbal responses without waiting for a delayed response prompt, and the present study followed this protocol. As shown above, conditions that required divided attention produced larger mean and peak pupil dilation than conditions that involved selective attention, even when the response period was included in the TEPR measurement window. Similar results regarding response period inclusion have recently been reported by Winn (2016), who found that differences in TEPRs between conditions were maintained and even amplified when the participant's response period was included in the TEPR measurement window. Thus, it may be appropriate to re-examine the protocol employed by some authors (Piquado et al, 2010; Zekveld et al, 2010; van Rijn et al, 2012; Koelewijn et al, 2014) that actively delays participants' responses, to exclude the response period from the TEPR measurement window.

In summary, the results of the current study confirm the conclusion of Koelewijn et al (2014) that the TEPR is specifically sensitive to divided auditory attention and demonstrate that this sensitivity is not an artefact of greater memory demand as it was maintained when the number of stimuli for recall was equal. The present study also demonstrates this sensitivity to divided auditory attention in a task with minimal linguistic load. Careful consideration of the cognitive load associated with divided auditory attention tasks may therefore be required during clinical audiological assessments.

Acknowledgments. We thank Bridget Fuller, Daniel Martino, and Abdulrahman Teia for assistance with data collection and performing the audiological testing and Dr. Tobias Loetscher and Dr. Shane Moon for loan of the pupillometry equipment. 


\section{REFERENCES}

American Speech-Language-Hearing Association (ASHA). (2005) (Central) Auditory Processing Disorders. http://www.asha.org/ policy/TR2005-00043/. Accessed April 4, 2015.

Aston-Jones G, Cohen JD. (2005) An integrative theory of locus coeruleus-norepinephrine function: adaptive gain and optimal performance. Annu Rev Neurosci 28:403-450.

Beatty J, Lucero-Wagoner B. (2000) The pupillary system. In: Berntson GG, ed. Handbook of Psychophysiology. 2nd ed. New York, NY: Cambridge University Press, 142-162.

Benarroch EE. (2009) The locus coeruleus norepinephrine system: functional organization and potential clinical significance. Neurology 73(20):1699-1704.

Bradshaw J. (1968) Pupil size and problem solving. Q J Exp Psychol A 20(2):116-122.

Bregman AS. (1994) Auditory Scene analysis: The Perceptual Organization of Sound. Cambridge, MA: MIT Press.

Broadbent DE. (1971) Decision and Stress. London, United Kingdom: Academic Press.

Bryden MP. (1988) An overview of the dichotic listening procedure and its relation to cerebral organization. In: Hugdahl KE, ed. Handbook of Dichotic Listening: Theory, Methods and Research. Suffolk, United Kingdom: John Wiley \& Sons, 1-45.

Cherry CE. (1953) Some experiments on the recognition of speech, with one and with two ears. J Acoust Soc Am 25(5):975-979.

Diamond A. (2013) Executive functions. Annu Rev Psychol 64:135-168.

Einhäuser W. (2017) The pupil as marker of cognitive processes. In: Zhao Q, ed. Computational and Cognitive Neuroscience of $\mathrm{Vi}$ sion. Singapore: Springer, 141-169.

Faul F, Erdfelder E, Lang AG, Buchner A. (2007) G*Power 3: a flexible statistical power analysis program for the social, behavioral, and biomedical sciences. Behav Res Methods 39:175-191.

Gilzenrat MS, Nieuwenhuis S, Jepma M, Cohen JD. (2010) Pupil diameter tracks changes in control state predicted by the adaptive gain theory of locus coeruleus function. Cogn Affect Behav Neurosci 10(2):252-269.

Hess EH, Polt JM. (1964) Pupil size in relation to mental activity during simple problem-solving. Science 143(3611):1190-1192.

Kahneman D, Beatty J. (1966) Pupil diameter and load on memory. Science 154(3756):1583-1585.

Kahneman D, Peavler WS, Onuska L. (1968) Effects of verbalization and incentive on the pupil response to mental activity. Can $J$ Psychol 22(3):186.

Kan A, Winn MB. (2016) Using pupillometry to investigate the better ear advantage. Poster session presented at: The 5th Joint Meeting of the Acoustical Society of America and Acoustical Society of Japan, November 28-December 2, Honolulu, HI.

Kimura D. (1961) Cerebral dominance and the perception of verbal stimuli. Can J Psychol 15(3):166.

Koelewijn T, Shinn-Cunningham BG, Zekveld AA, Kramer SE. (2014) The pupil response is sensitive to divided attention during speech processing. Hear Res 312:114-120.

Koelewijn T, Zekveld AA, Festen JM, Kramer SE. (2012) Pupil dilation uncovers extra listening effort in the presence of a singletalker masker. Ear Hear 33(2):291-300.
Kramer SE, Kapteyn TS, Festen JM, Kuik DJ. (1997) Assessing aspects of auditory hangicap by means of pupil dilation. Int $J$ Audiol 36(3):155-164.

Kramer SE, Lorens A, Coninx F, Zekveld AA, Piotrowska A, Skarzynski H. (2013) Processing load during listening: the influence of task characteristics on the pupil response. Lang Cogn Process 28(4):426-442.

Kuchinsky SE, Ahlstrom JB, Vaden KI, Cute SL, Humes LE, Dubno JR, Eckert MA. (2013) Pupil size varies with word listening and response selection difficulty in older adults with hearing loss. Psychophysiology 50(1):23-34.

Martin NA, Brownell R. (2008) TAPS-3: Test of Auditory Processing Skills. 3rd ed. Northumberland, United Kingdom: Ann Arbor Publishers.

Mattys SL, Davis MH, Bradlow AR, Scott SK. (2012) Speech recognition in adverse conditions: a review. Lang Cogn Process 27(7-8):953-978.

McGarrigle R, Munro KJ, Dawes P, Stewart AJ, Moore DR, Barry JG, Amitay S. (2014) Listening effort and fatigue: what exactly are we measuring? A British Society of Audiology Cognition in Hearing Special Interest Group 'white paper'. Int J Audiol 53(7):433-445.

Moore DR. (2015) Sources of pathology underlying listening disorders in children. Int $J$ Psychophysiol 95(2):125-134.

Musiek FE. (1983) Assessment of central auditory dysfunction: the dichotic digit test revisited. Ear Hear 4(2):79-83.

Parkinson SR. (1974) Variability and control in dichotic memory. J Exp Psychol 102(1):67.

Piquado T, Isaacowitz D, Wingfield A. (2010) Pupillometry as a measure of cognitive effort in younger and older adults. Psychophysiology 47(3):560-569.

Schow RL, Seikel JA, Chermak GD, Berent M. (2000) Central auditory processes and test measures: ASHA 1996 revisited. Am $J$ Audiol 9(2):63-68.

Simpson H. (1969) Effects of a task-relevant response on pupil size. Psychophysiol 6(2):115-121.

Simpson H, Hale SM. (1969) Pupillary changes during a decisionmaking task. Percept Mot Skills 29(2):495-498.

Sirois S, Brisson J. (2014) Pupillometry. Wiley Interdiscip Rev Cogn Sci 5(6):679-692.

Tomlin D, Dillon H, Sharma M, Rance G. (2015) The impact of auditory processing and cognitive abilities in children. Ear Hear 36(5):527-542.

van Rijn H, Dalenberg JR, Borst JP, Sprenger SA. (2012) Pupil dilation co-varies with memory strength of individual traces in a delayed response paired associate task. PLoS One 7(12):1-8.

Winn MB. (2016) Rapid release from listening effort resulting from semantic context, and effects of spectral degradation and cochlear implants. Trends Hear 20:1-17.

Winn MB, Edwards JR, Litovsky RY. (2015) The impact of auditory spectral resolution on listening effort revealed by pupil dilation. Ear Hear 36(4):e153-e165.

Zekveld AA, Kramer SE, Festen JM. (2010) Pupil response as an indication of effortful listening: the influence of sentence intelligibility. Ear Hear 31(4):480-490.

Zekveld AA, Kramer SE, Festen JM. (2011) Cognitive load during speech perception in noise: the influence of age, hearing loss, and cognition on the pupil response. Ear Hear 32(4):498-510. 